\title{
Influencia de la sombra y fertilización en la resistencia de clones élites Coffea arabica L. ante la incidencia y severidad de Hemileia vastatrix Berk. \& Br.
}

\section{Influence of shade and fertilization on the resistance of elite clones of Coffea arabica $\mathrm{L}$. to the incidence and severity of Hemileia vastatrix Berk. \& Br.}

\author{
Amparito Huaman
}

\begin{abstract}
RESUMEN
La presente investigación tuvo como objetivo evaluar la influencia de la sombra y fertilización en la resistencia de clones élites de café (Coffea arabica L.) ante la incidencia y severidad de Hemileia vastatrix Berk. \& Br., Rodríguez de Mendoza, Amazonas, Perú. Se instaló bajo un diseño en bloque completo al azar (DBCA) con arreglo factorial, con dos factores; el primero porcentajes de sombra $(0 \%, 50 \%$ y $80 \%)$; el segundo factor, dosis de fertilizante orgánico guano de isla y roca fosfórica en proporción $2: 1 \mathrm{v} / \mathrm{v}(0,4,8$ y $12 \mathrm{~g})$, dando un total de 12 tratamientos, 3 bloques y 36 unidades experimentales. Se utilizaron plantas clonadas de 4 meses de edad, obtenidas a partir de germoplasmas en jardines clonales. Las plantas clonales fueron inoculadas con una disolución de uredosporas de roya amarilla (Hemileia vastatrix), colectadas de fincas cafetaleras del distrito de Huambo, evaluándose la incidencia, severidad y área bajo la curva del progreso de la enfermedad. Se encontró que las plantas sin sombra presentaron baja incidencia y severidad ( $0.02 \%$ y $0.03 \%$ respectivamente), no obstante, a medida que el porcentaje de sombra se incrementa la incidencia y severidad fue mayor. Las dosis de fertilizante presentaron diferencias significativas sobresaliendo los tratamientos con (8 y $12 \mathrm{~g})$, así mismo las plantas no fertilizadas presentaron altos porcentajes y mayor infección. El efecto de sombras y dosis de fertilizante fueron relevantes, determinando que a medida la sombra disminuye el porcentaje de infección del hongo disminuye a medida que la dosis de fertilizante es mayor el porcentaje de infección disminuye.
\end{abstract}

Palabras clave: Fertilizante, inoculación, roya amarilla, uredosporas

\begin{abstract}
The objective of this research was to evaluate the influence of shade and fertilization on the resistance of elite coffee clones (Coffea arabica L.) to the incidence and severity of Hemileia vastatrix Berk. \& Br., Rodríguez de Mendoza, Amazonas, Peru. It was installed under a randomized complete block design (DBCA) with factorial arrangement, with two factors; the first percentages of shade $(0 \%, 50 \%$ and $80 \%)$; the second factor, dose of organic guano island fertilizer and phosphoric rock in a ratio of $2: 1 \mathrm{v} / \mathrm{v}(0,4,8$ and $12 \mathrm{~g})$, giving a total of 12 treatments, 3 blocks and 36 experimental units. 4-month-old cloned plants were used, obtained from germplasm in clonal gardens. The clonal plants were inoculated with a solution of yellow rust (Hemileia vastatrix) uredospores, collected from coffee farms in the Huambo district, evaluating the incidence, severity and area under the curve of disease progress. It was found that the plants without shade presented low incidence and severity $(0.02 \%$ and $0.03 \%$ respectively), however, as the percentage of shade increases, the incidence and severity was higher. Fertilizer doses showed significant differences, highlighting treatments with ( 8 and $12 \mathrm{~g}$ ), likewise unfertilized plants presented high percentages and higher infection. The effect of shadows and fertilizer dose were relevant, determined that as the shadow decreases, the percentage of infection of the fungus decreases as the dose of fertilizer is greater, the percentage of infection decreases.
\end{abstract}

Keywords: Fertilizer, inoculation, yellow rust, uredospores

${ }^{1}$ Bachiller de la Facultad de Ingeniería y Ciencias Agrarias. Universidad Nacional Toribio Rodríguez de Mendoza de Amazonas. Correo electrónico: 7057569113@untrm.edu.pe, ahuamanpilco@gmail.com 


\section{INTRODUCCIÓN}

La Región Amazonas, Perú, es la tercera en producción de café a nivel nacional por detrás de Junín y Cuzco. Las principales zonas de producción son la provincia de Rodríguez de Mendoza, Bagua, Utcubamba. La superficie estimada de cultivo de café en Rodríguez de Mendoza es de 5668 Has (Quiroz, 2015).

La roya (Hemileia vastatrix) del café es una enfermedad que limita seriamente la producción de Coffea arabica; su presencia está determinada por las condiciones climáticas, ya que una alta humedad es necesaria para la esporulación y dispersión de las esporas. Dado que la roya es un patógeno biotrófico se ve favorecida por el retraso en la senescencia de las hojas, por ello se podría decir que la fertilización tiene gran influencia ante el ataque de este patógeno.

El fósforo puede influir en la resistencia fisiológica en dos vías: incentiva el funcionamiento normal de la célula lo cual influye en el suministro de alimento para los biotróficos; pero también aumenta las defensas de las plantas. Las deficiencias de potasio se ven relacionadas con mayor ataque de parásitos biotróficos lo contrario ocurre en niveles adecuados (Walters \& Bingham, 2007). No obstante, existen estudios que concluyen que los parásitos biotróficos se favorecen de altas concentraciones de $\mathrm{N}$ en la planta(Avelino, Hoopen, \& DeClerck, 2011). Esto a su vez concuerda con lo encontrado por (Lagos, 2014), en Honduras donde, hubo mayor incidencia de roya en parcelas donde se aplicaron mayores cantidades de $\mathrm{N}$ al suelo.

Segura (2016) menciona que existe mayor lavado de las esporas en los tratamientos a pleno sol, respecto a los de sombra. Lo anterior indica que se conservan más esporas bajo sombra ya que ésta intercepta el agua y al haber menos agua en la plantación se produce menor lavado comparado con condiciones a pleno sol.

La finalidad de esta investigación fue contribuir a entender la influencia de la sombra y fertilización en la resistencia de clones elites de café a la roya amarilla $\boldsymbol{H}$. vastatrix; además se proporcionó a los productores de café de la provincia de Rodríguez de Mendoza y la región Amazonas, mayores criterios para el manejo de la nutrición de plántulas clones de café durante la etapa de vivero en lo que a dosis y fuentes se refiere, y el porcentaje de sombra adecuado para contrarrestar el daño de la enfermedad.

\section{MATERIALES Y MÉTODOS}

\section{Selección de plantas}

Se contó con plantas de café de las cuales se tuvo que seleccionar 144 plantas para su uso en la investi- gación, dicha selección consistió en determinar plantas en buen estado, es decir aquellas que no presenten enfermedades que pudieran intervenir en el estudio.

\section{Limpieza del área}

La limpieza del área donde se instaló el trabajo, se realizó manualmente haciendo uso de algunas herramientas tales como machete y lampa para eliminar las malezas que se encontraron en el área. Luego se procedió a aplanar y agregar arena, para obtener una plataforma o base homogénea para el asentamiento de las bolsas que contenían a las plantas de café.

\section{Instalación de sombra}

Una vez realizada la plataforma, y realizado las mediciones de acuerdo al diseño experimental se procedió a colocar 24 soportes de madera con dimensiones $70 \mathrm{~cm}$ de altura, $12 \mathrm{de} 1.20 \mathrm{~cm}$, y $12 \mathrm{de}$ $30 \mathrm{~cm}$, distribuyéndose según el diseño del experimento. Luego se colocó mallas raschell con porcentajes de $50 \%$ y $80 \%$ de sombra, según la distribución de los factores en estudio.

\section{Preparación del fertilizante}

El fertilizante a utilizar fue la mezcla de guano de isla y roca fosfórica en proporción $2: 1 \mathrm{v} / \mathrm{v}$ respectivamente. Lo primero que se realizó fue elegir un recipiente con un volumen establecido luego se procedió a medir los fertilizantes guano de isla, roca fosfórica y se realizó la mezcla de acuerdo a la proporción.

\section{Aplicación de la fertilizante a plantas de café}

La fertilización se realizó posterior al colocado de sombras. Para ello se fabricó etiquetas y se colocó en una planta representativa de todos los tratamientos, la identificación con sus dosis respectivas en estudio. Primero se pesó las dosis en una balanza de precisión, luego se realizó 3 hoyos en forma de triángulo alrededor de la bolsa y se procedió a colocar el fertilizante sobre la superficie del sustrato, teniendo cuidado que no entre en contacto directo con las plantas para evitar riesgos de mortandad por quemazón o toxicidad (Fundación Salvadoreña para Investigaciones del Café , 2008), dejando así que el fertilizante se ubique aproximadamente en el borde de la bolsa (CICAFE, 2011). El fertilizante fue fraccionado en las siguientes dosis: 4,8 y $12 \mathrm{~g} /$ planta, así mismo se dejó un testigo sin fertilizante $(0 \mathrm{~g})$.

\section{Colección de la fuente de inóculo de la roya amarilla (Esporas de roya)}

La roya amarilla fue obtenida de hojas infectadas por dicha enfermedad, de preferencia se colectó hojas de café de la variedad típica, para ello se desarrolló la siguiente metodología. Se cortó las hojas infectadas y se colocó en un depósito limpio y hermético. Para esta 
colecta se tuvo en cuenta que las plantas de café estuvieron libres de fungicida, tanto en las plantas de las parcelas en estudio como de parcelas adyacentes (Vallejos, 2016).

En laboratorio, con la ayuda de un estereoscopio se procedió a observar las hojas colectadas. Aquellas partes de las hojas que presentaron otros hongos fueron eliminadas con una tijera y las uredosporas libres de endoparásitos fueron pasadas a un taper grande.

\section{Preparación del inóculo de H. vastatrix}

Se preparó, utilizando la metodología de (Vallejos, 2016), de la siguiente manera: se disolvió en un litro de agua destilada las hojas infectas con las esporas de roya (esporas maduras), luego se procedió a mover la solución con la finalidad de homogenizar la concentración de las uredosporas. La preparación del inóculo se realizó teniendo previsto la infestación instantánea a los plantones, para acreditar la infección. Se realizó un conteo de las esporas, para ello, se tomó con la micropipeta una cantidad de la mezcla preparada, se colocó un cubre objetos sobre la cámara de Neubauer y se precedió a introducir la muestra o solución en la cámara de Neubauer. La cámara Neubauer con la muestra se enfocó al microscopio para el conteo de las uredosporas de roya. El conteo se hizo en 9 cuadros grandes, donde se pudo observar 2 esporas por cuadro. Con la finalidad de conocer la cantidad de uredosporas infestadas se realizó el conteo, haciendo un total de 20000 uredosporas $/ \mathrm{ml}$. Utilizando la fórmula para conteo de cuadros grandes(Vallejos, 2016).

$$
\text { Concentración }=\frac{\text { Número de células } * 10000}{\text { Número de cuadros }}
$$

\section{Infestación con roya a las plantas élites de café clonado}

Las plantas de café típica propagadas vegetativamente, fueron inoculadas con la dilución de uredosporas descritas anteriormente en horas de la tarde cuando los rayos solares se ocultaron, previamente las plantas de café fueron irrigadas antes de la inoculación. Para ello se usó un aspersor manual, distribuyendo sobre las hojas de todas las plantas, la aplicación pulverizada se realizó de forma uniforme. Finalmente, las plantas que fueron inoculadas con uredosporas de roya amarilla se cubrió las hojas con una bolsa de plástico húmeda y se cerró; se colocó una hoja de periódico sobre la bolsa de plástico para evitar la luz solar directa sobre las hojas inoculadas (para evitar la inhibición de la germinación de las esporas); 24 horas después de la inoculación, se procedió a retirar la bolsa de plástico, pero se guardó la hoja de periódico sobre las hojas inoculadas; después de 24 horas, se retiró la hoja del periódico y dejo libre las plantas de café para su posterior evaluación(Varzea, 2016).

\section{Evaluación de Variables}

Determinación de la incidencia de la roya $(H$. vastatrix) del café

Para la evaluación se tomó los siguientes datos:

1.Número de hojas presentes al momento de cada lectura (NHP).

\section{Número de hojas afectadas por la roya (NHA).}

Incidencia de la roya: El porcentaje de hojas afectadas por la roya o incidencia, se calculó mediante la relación del número de hojas afectadas (NHA) y el número de hojas presentes (NHP) al momento de la evaluación (Alvarado \& Solórzano, 2001)

$$
\% \text { de incidencia }=\frac{N H A}{N H P} * 100
$$

\section{Determinación de la severidad de roya $(H$. vastatrix) del café}

En cada planta se determinó el número de hojas y los síntomas observados determinaron el grado de severidad de la enfermedad, según el porcentaje de daño causado en la hoja. Para evaluar severidad en planta se utilizó el procedimiento recomendado por la institución SENASA de Perú del 2012 lo cual se presenta a continuación(Garcia , 2013).

Tabla 1

Escala de severidad para roya

\begin{tabular}{cl}
\hline $\begin{array}{c}\text { Grado o } \\
\text { calificación }\end{array}$ & \multicolumn{1}{c}{ Descripción } \\
\hline 0 & $\begin{array}{l}\text { Sano o sin síntomas visibles } \\
\text { Síntomas visibles llegando de 1 a } \\
5 \% \text { del área total sana }\end{array}$ \\
2 & $\begin{array}{l}\text { Las manchas empiezan a unirse, } \\
\text { llegando a ocupar del } 6 \text { al 20\%de] } \\
\text { área sana } \\
\text { Las hojas comienzan a necrosarse } \\
\text { de manera muy notoria, afectando } \\
\text { del 21 al 50\% del aérea sana } \\
\text { Mayor al 50 del área foliar se } \\
\text { encuentra afectada }\end{array}$ \\
\hline
\end{tabular}

Fuente: (SENASA, 2012). 


\section{Figura 1}

Grado de la escala de severidad (SENASA, 2012).

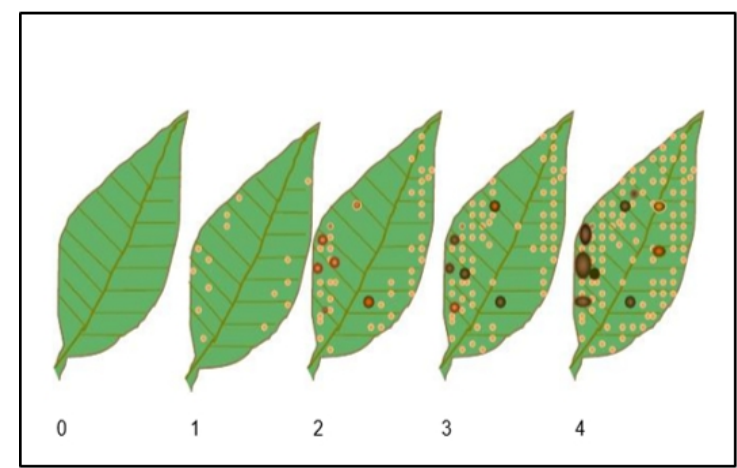

La determinación de severidad de la roya del café se hizo con la fórmula:

$$
S E V=\frac{(N 0 * 0)+(N 1 * 1)+(N 2 * 2)+(N 3 * 3)+(N 4 * 4)}{N * 4}
$$

\section{Dónde:}

N0 $=\#$ Hojas con valor 0 de la escala.

N1 = \# Hojas con valor 1 de la escala.

$\mathrm{N} 2=\#$ Hojas con valor 2 de la escala.

N3 =\# Hojas con valor 3 de la escala.

N4 = \# Hojas con valor 4 de la escala

Determinación del Área bajo la curva de progreso de la enfermedad (ABCPE)

La determinación del área bajo la curva del progreso de la enfermedad se realizó mediante la fórmula propuesta por (Gamarra, Torres, Casas, \& Riveros, 2015).

$$
A B C P E=\sum_{i=1}^{n}[X i+1+X i / 2][T i+1-T i]
$$

\section{Dónde:}

$\mathrm{X}_{\mathrm{i}}=$ Incidencia de la enfermedad en un tiempo dado de observación.

$\mathrm{X}_{\mathrm{i}+1}=$ Porcentaje de daño del follaje en el día $\mathrm{T}+1$.

$\mathrm{T}=$ Tiempo en días.

$\left(\mathrm{T}_{\mathrm{i}+1}-\mathrm{T}_{\mathrm{i}}\right)=$ Número de días resultado de la diferencia entre la primera y segunda lectura.

$\mathrm{N}=$ Número total de observaciones.

La severidad e intensidad de la enfermedad fue evaluada cada 15 días en el periodo de desarrollo de la roya amarilla del café (Hemileia vastatrix).

\section{RESULTADOS}

\section{Tabla 2}

Análisis de Varianza (ANOVA) para la variable incidencia de la roya amarilla (H. vastatrix), con porcentajes de sombra y dosis de fertilizante

\begin{tabular}{lccccc}
\hline F.V. & SC & gl & CM & F & p-valor \\
\hline $\begin{array}{l}\text { SOMBRA } \\
\text { DOSIS DE }\end{array}$ & 0.61 & 2 & 0.3 & 62. & 0.0000 \\
$\begin{array}{l}\text { FERTILIZ } \\
\text { ANTE }\end{array}$ & 0.07 & 3 & 0.02 & 4.75 & 0.0106 \\
$\begin{array}{l}\text { BLOQUE } \\
\text { SOMBRA* }\end{array}$ & 0.02 & 2 & 0.01 & 2.43 & 0.1110 \\
$\begin{array}{l}\text { DOSIS DE } \\
\text { FERTILIZ }\end{array}$ & 0.07 & 6 & 0.01 & 2.31 & 0.0699 \\
$\begin{array}{l}\text { AN. } \\
\text { Error }\end{array}$ & 0.11 & 22 & 0 & & \\
Total & 0.87 & 35 & & & \\
\hline
\end{tabular}

*=significativo; SC: suma de cuadrados; gl: grados de libertad; CM: cuadrados medios; F: factor $F$

El análisis de varianza (ANOVA), reporta que en el factor sombra existe diferencia estadística significativa en el \% de incidencia de la roya amarilla. En cuanto a la dosis de fertilizante, se observó que para la variable \% de incidencia hay diferencia estadística.

\section{Figura 2}

Prueba de Tukey $(P<0,05)$ para la incidencia de la roya del café con porcentajes de sombra

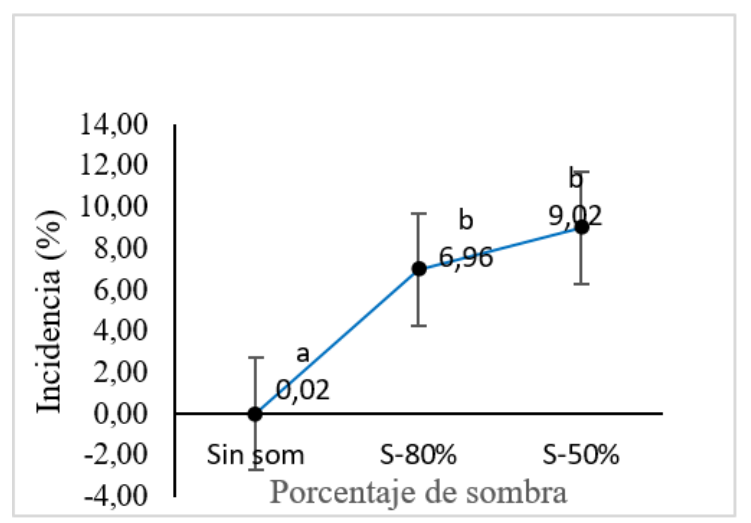

se observa que la incidencia de la roya amarilla $\boldsymbol{H}$. vastatrix del café, estuvo fuertemente influenciado por el $50 \%$ y $80 \%$ de sombra, donde se reportaron medias de 9.02 y 6.96; comparado con el factor sin sombra, presentando este último una media de 0.02 . 


\section{Figura 3}

Prueba de Tukey $(P<0,05)$ para la incidencia de la roya del café con dosis de fertilizante.

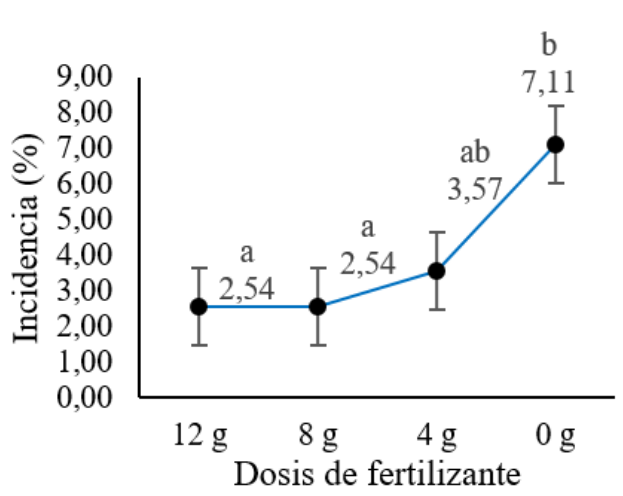

Se observa que la incidencia de la roya amarilla $\boldsymbol{H}$. vastatrix del café, estuvo fuertemente influenciado por el factor $0 \mathrm{~g} /$ planta de fertilizante, donde se reportaron medias de 7.11 , seguido de una dosis de $4 \mathrm{~g}$ que presenta medias de 3.57, mientras que el factor $12 \mathrm{~g}$ y $8 \mathrm{~g}$ tuvo influencia positiva, presentando estos últimos una media de 2.54. Es decir, las plantas sin fertilizante presentaron mayor incidencia de roya en comparación a los fertilizados con dosis de 4, 8 y $12 \mathrm{~g} /$ planta de fertilizante.

\section{Tabla3}

Análisis de Varianza (ANOVA) para la variable severidad de la roya amarilla (H. vastatrix), con porcentajes de sombray dosis de fertilizante.

\begin{tabular}{llllll}
\hline F.V. & SC & gl & CM & F & p-valor \\
\hline SOMBRA & 98.92 & 2 & 49.46 & 11.76 & $0.0003^{*}$ \\
DOSIS DE & 60.44 & 3 & 20.15 & 4.79 & 0.0102 \\
FERTILIZANTE & & & & & \\
BLOQUE & 15.44 & 2 & 7.72 & 1.84 & 0.1831 \\
SOMBRA*DOSIS & & & & & \\
DE & 49.09 & 6 & 8.18 & 1.95 & 0.118 \\
FERTILIZAN. & & & & & \\
Error & 92.52 & 22 & 4.21 & & \\
Total & 316.41 & 35 & & & \\
\hline
\end{tabular}

*=significativo; SC: suma de cuadrados; gl: grados de libertad; CM: cuadrados medios; F : factor F

El análisis de varianza (ANOVA), reporta que existe diferencia estadística entre porcentajes de sombra. En cuanto a la dosis de fertilizante, se observó que para la variable severidad si existe diferencia estadística.

\section{Figura 4}

Prueba de Tukey $(P<0,05)$ para la severidad de la roya del café con porcentajes de sombra

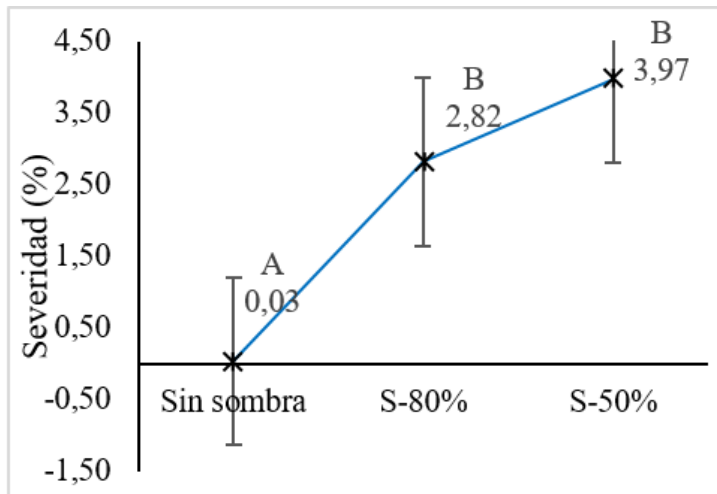

Porcentaje de sombra

Se observa que el factor sin sombra presentó influencia positiva en el desarrollo de la severidad, reportando un promedio de 0.03; así mismo se observa que el $50 \%$ y $80 \%$ de sombra presentó influencia negativa en la severidad de la roya.

\section{Figura 5}

Prueba de Tukey $(P<0,05)$ para la severidad de la roya del café con dosis de fertilizante.

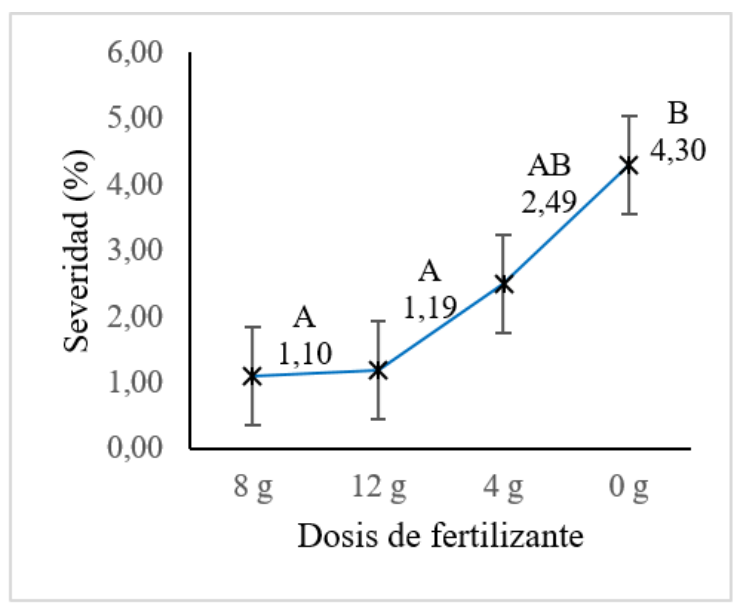

Se presenta la comparación de medias para la variable severidad de la roya del café, observando que el factor $8 \mathrm{~g}$ y $12 \mathrm{~g} /$ planta de fertilizante no presentan diferencias estadísticas significativas entre sí, por lo que, para esta variable, mostraron una influencia positiva en el desarrollo de la severidad de la roya, con un promedio de 1,19 y 1,10 respectivamente, del mismo modo se aprecia la severidad de la roya sin fertilizante fue mayor que los demás. 


\section{Tabla4}

Análisis de Varianza (ANOVA) para el ABCDE de la roya amarilla (H. vastatrix), con porcentajes de sombray dosis de fertilizante.

\begin{tabular}{llllll}
\hline F.V. & SC & gl & CM & F & $\begin{array}{l}\text { p- } \\
\text { valor }\end{array}$ \\
\hline SOMBRA & 2498628.31 & 2 & $\begin{array}{l}12493 \\
14.15\end{array}$ & 13.4 & $\begin{array}{l}0.000 \\
2^{*}\end{array}$ \\
$\begin{array}{llllll}\text { DOSIS DE } \\
\text { FERTILIZ }\end{array}$ & 1410251.49 & 3 & 47008 & 5.83 & 0.008 \\
ANTE & & & & & $3 *$ \\
BLOQUE & 396746.05 & 2 & 19837 & 2.13 & 0.142 \\
SOMBRA & & & 3.02 & & 9 \\
*DOSIS & & & 18978 & 2.04 & 0.103 \\
$\begin{array}{l}\text { DE } \\
\text { FERTILIZ }\end{array}$ & 1138711.31 & 6 & 5.22 & & 5 \\
AN. & & & & & \\
Error & 2050456.33 & 22 & 93202 & & \\
Total & 7494793.49 & 35 & & & \\
\hline
\end{tabular}

*=significativo; SC: suma de cuadrados; gl: grados de libertad; CM: cuadrados medios; F: factor $F$

El análisis de varianza (ANOVA), reporta que existe diferencia estadística entre los porcentajes de sombra. En cuanto a la dosis de fertilizante, se observó que para la variable Área bajo la curva de desarrollo de la enfermedad (ABCDE) si hay diferencia estadística.

\section{Figura 6}

Prueba de Tukey $(P<0,05)$ para el $A B C D E$ de la roya del café con porcentajes de sombra.

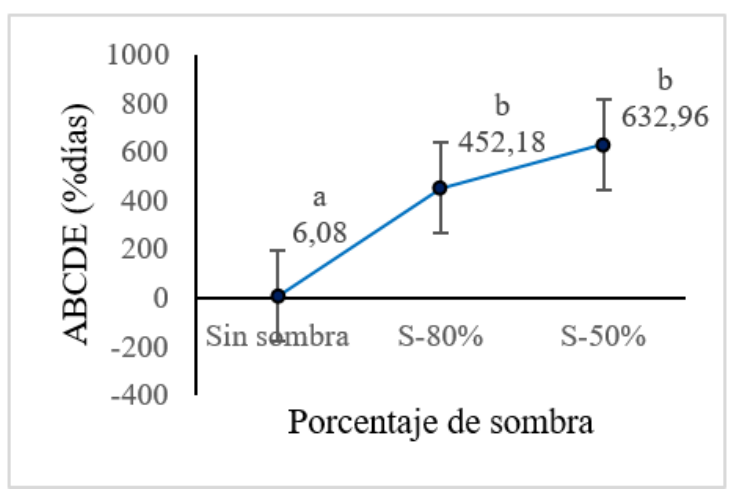

Se presenta la comparación de medias para la variable área bajo la curva de desarrollo de la roya del café, observando que el factor sin sombra presentó influencia positiva en el desarrollo de la severidad y por consiguiente menor área bajo la curva de desarrollo de la enfermedad, reportando un promedio de 6.08 \%días, así mismo observamos que al 50 y $80 \%$ de sombra no presentan diferencias estadísticas significativas entre sí.

\section{Figura 7}

Prueba de Tukey $(P<0,05)$ para el ABCDE de la roya del caféc con dosis de fertilizante.

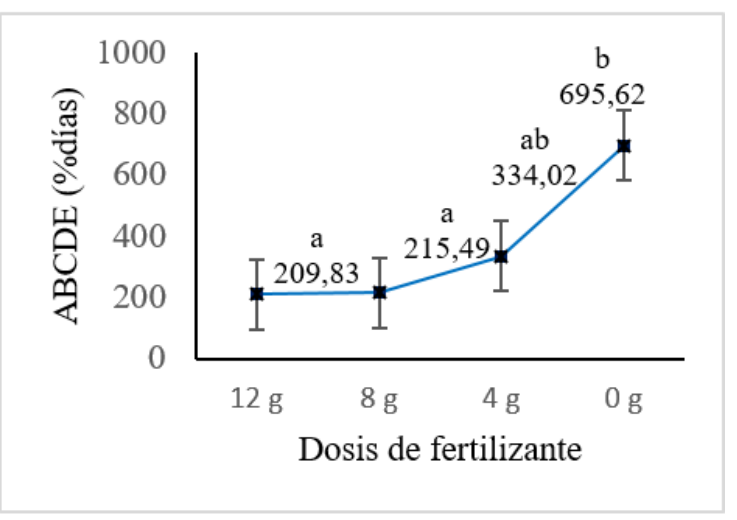

Se presenta la comparación de medias para la variable área bajo la curva de desarrollo de $\boldsymbol{H}$. vastatrix del café, observando que la dosis $0 \mathrm{~g} /$ planta de fertilizante presento influencia negativa, donde se reportaron medias de 695,62\%días, comparado con una dosis de 8 y $12 \mathrm{~g}$ respectivamente que no presentan diferencias estadísticas significativas entre sí, presentando estos últimos una media de 215,49 y 209,83\% días.

\section{DISCUSIÓN}

Al evaluar la influencia de la sombra y fertilización en la resistencia de clones élites de café (Coffea arabica L.) ante la incidencia y severidad de Hemileia vastatrix Berk. \& Br., en la provincia de Rodríguez de Mendoza, Amazonas, Perú, se observa que las plantas elites que recibieron mayor dosis de fertilización presentaron menor incidencia y severidad de la roya amarilla del café, esto se corrobora con lo mencionado por Avelino et al., (2006), quien encontró que fincas bien fertilizadas o fincas en suelos fértiles eran menos expuestas a ataques severos de la roya.

Dado que la roya es un patógeno biotrófico se ve favorecida por el retraso en la senescencia de las hojas, por lo cual el nitrógeno podría tener efectos favorables (Walters \& Bingham, 2007). En nuestro estudio las plantas que fueron fertilizadas con mayor dosis de fertilizante ( 8 y $12 \mathrm{~g}$ ), fueron más resistentes al ataque de la roya amarilla. Estas dosis aportaron bastante Nitrógeno contenido en el guano de isla. Lo que contradice a lo dicho por los autores y por Lagos (2014), quien en su investigación "Efecto de la condición química del suelo y de la fertilización sobre la incidencia, severidad y resistencia fisiológica de plantas de café a la roya (Hemileia vastatrix)", encontró que la incidencia de la roya fue favorecida por mayores cantidades de nitrógeno aplicado al suelo y urea aplicada al follaje.

En nuestro estudio las plantas que fueron fertilizadas 
con mayor dosis de roca fosfórica (8 y $12 \mathrm{~g}$ ) fueron más resistentes al ataque de la roya amarilla, esto se corrobora con lo mencionado por Walters y Bingham (2007), que sostienen que el fósforo puede influir en la resistencia fisiológica en dos vías: incentiva el funcionamiento normal de la célula lo cual influye en el suministro de alimento para los biotróficos; pero también aumenta las defensas de las plantas y también las mayores severidades se asociaron a menor cantidad de fósforo aplicado al suelo y mayor cantidad de urea aplicada al follaje (Lagos, 2014).

Savary et al., (1995) encontró que plantas de arroz con altos niveles de nitrógeno tenían mayores ataques de Rhizoctonia solani (tizón de la vaina del arroz), en nuestro estudio las plantas que fueron fertilizadas con mayor dosis de fertilizante y por consiguiente mayores niveles de nitrógeno tuvieron menor ataque de la roya amarilla del café. Por otro lado, Dordas (2008) y Avelino et al., (2011), establecieron que los parásitos biotróficos se favorecen de altas concentraciones de $\mathrm{N}$ en la planta. Esto concuerda con lo encontrado por Lagos (2014), en Honduras donde hubo mayor incidencia de roya en parcelas donde se aplicaron mayores cantidades de $\mathrm{N}$ al suelo.

En este estudio al evaluar la influencia de la sombra y fertilización en la resistencia de clones élites de café (Coffea arabica L.) ante la incidencia y severidad de Hemileia vastatrix Berk. \& Br., se observa que las plantas elites que se encontraron bajo $50 \%$ y $80 \%$ de sombra presentaron mayor incidencia y severidad de la roya amarilla del café, esto se corrobora con López et al., (2012) quién encontró mayor incidencia de roya bajo sombra en plantas con cargas fructíferas controladas. Esto sugiere que la sombra favoreció los procesos pre-infecciosos de germinación y penetración, los cuales determinan la incidencia, respecto al pleno sol.

López (2010), encontró relación positiva entre la sombra y la incidencia de la roya cuando la carga productiva era homogenizada. Las máximas infecciones de roya se dieron en porcentajes de sombra entre $35 \%$ y $73 \%$. En nuestra investigación las mayores infecciones de la roya se dieron a $50 \%$ de sombra lo que concuerda con lo mencionado por el autor.

En nuestra investigación los clones de café bajo 50\% y $80 \%$ de sombra alcanzaron mayor incidencia y severidad de la roya, lo cual indica que la sombra favorece el desarrollo de la enfermedad, esto se corrobora por estudios de Brenes (2016), sobre los procesos de colonización, esporulación y dispersión aérea de la roya (Hemileia vastatrix) sobre plantas de café (Coffea arabica), en la zona de Turrialba, Costa Rica, quien en estudios complementarios, estableció que bajo sombra de cashá (Chloroleucon eurycyclum), cuando llueve, se favorece el proceso de dispersión, especialmente durante la noche.
Esto concuerda con lo encontrado por Boudrot et al., 2016, quien establece cómo la sombra en los días con lluvia favorece la dispersión de las uredosporas, ya que el agua se acumula en las hojas del árbol de sombra, formando gotas de agua, las cuales caen con más fuerza impactando las hojas y liberando las uredosporas que se encuentran en el envés. Por el contrario, en días secos, Boudrot et al., (2016) y Brenes (2016) mencionan que la sombra reduce la dispersión de las uredosporas debido a que los árboles interceptan el viento, el cual es el que libera y transporta las uredosporas en ausencia de lluvia.

\section{CONCLUSIONES}

Los porcentajes de sombra y las dosis de fertilizante presentaron influencia en la resistencia de clones de café variedad típica, los tratamientos con $50 \%$ y $80 \%$ de sombra tuvieron influencia negativa disminuyendo la resistencia de los clones de café, mientras que el tratamiento bajo $0 \%$ de sombra fue el tratamiento que presentó influencia positiva aumentando la resistencia. En cuanto a la fertilización plantas bien fertilizadas $(8 \mathrm{~g}$ y $12 \mathrm{~g}$ de fertilizante) son más resistentes a la roya, mientras que plantas $\sin$ fertilizar ( $0 \mathrm{~g}$ de fertilizante) son menos resistentes.

El porcentaje de sombra que tuvo influencia negativa en la incidencia y severidad de la roya amarilla del café fue el 50\% y $80 \%$ de sombra, mientras que un $0 \%$ de sombra presento influencia positiva disminuyendo la incidencia y severidad de la roya. Lo cual indica que a las condiciones de sombra el café es más susceptible a la enfermedad.

Las dosis de fertilización que tuvo influencia positiva en el desarrollo de la incidencia y severidad de la roya amarilla del café fueron de $8 \mathrm{~g}$ y $12 \mathrm{~g}$ de fertilizante presentando menor desarrollo de la enfermedad, mientras que una dosis de $0 \mathrm{~g}$ de fertilizante presentó influencia negativa aumentando la incidencia y severidad de la roya. Lo cual indica que a mayor dosis de fertilización o plantas bien fertilizadas son más resistentes a la enfermedad.

\section{REFERENCIAS BIBLIOGRÁFICAS}

Avelino, J., Hoopen, G., \& DeClerck, F. (2011). Ecological Mechanisms for Pest and Disease Control in Coffee and Cacao Agroecosystems of the Neotropics. Measuring ecosystem services, 92-118.

Cicafe. (2011). Guía técnica para el cultivo del cafe. Costa Rica: Instituto del Café en Costa Rica.

Fundación Salvadoreña para Investigaciones del Café . (23 de Julio de 2008). https://www.en 
gormix.com/agricultura/articulos/fertilizacio n-cafeto-t27565.htm. Obtenido de https:// www.engormix. com/agricultura/articulos /fertilizacion-cafeto-t27565.htm.

Lagos, S. (2014). Efecto de la condicion química del suelo y de la fertilizacion sobre la incidencia, severidad y resistemcia fisiológica de plantas de café a la roya (Hemileia vastatrix). Turrialba, Costa Rica: Centro Agronómico Tropical de Investigacion y Enseñanza.

Quiroz, F. (25 de Octubre de 2015). Difunden café de Amazonas en sus diversas presentaciones en "Expo Café 2015". Andina agencia peruana de noticias.

Segura, B. (2016). Efecto de la sombra en el cultivo del café sobre los procesos de esporulación, la dispersión a través de agua y la deposición, de Hemileia vastatrix, en Turrialba, Costa Rica. Catie.

Vallejos, J. (2016). Resistencia de plantas café arábico (Coffea arabica) variedad "Caturra Roja" a la Roya Amarila (Hemilea vastatrix), en la Region San Martín. Tarapoto: Universidad Nacional de San Martín.

Varzea, V. (2016). Protocolo para enviar muestras de roya del café a CIFC. Centro de Investigación de Ferrugens do Cafeeiro (Cifc)

Walters, D., \& Bingham, I. (2007). Influence of nutrition on disease development caused by fungal pathogens: implications for plant disease control. Ann Appl Biol, 11. 